Primary care

\section{Primary care ophthalmology}

\section{R Smith}

What? where? who? why?

$\mathrm{T}$ here was once a time when "primary care" was synonymous with general practice, "secondary care" with district general hospitals, "tertiary care" with teaching hospitals, and everyone who worked in the National Health Service knew their place. Now everything seems so confusing. Is the teaching hospital consultant who conducts an outreach clinic in a general practice providing primary, secondary, or tertiary care? What about the general practitioner (GP) with a specialist interest who performs argon laser photocoagulation for diabetic retinopathy in his own practice premises? And what of mobile cataract surgical teams?

The truth is that the nomenclature of primary, secondary, and tertiary care has never served ophthalmology particularly well. Most GPs learn little ophthalmology as undergraduates or during GP vocational training and few practices have more than the most basic equipment for ophthalmic examination. Although eye problems present commonly in general practice and many GPs are willing to treat a limited range of minor eye disorders, most hospital eye departments continue to be major providers of first-line ophthalmic care for the simplest to the most complex eye conditions. ${ }^{1}$ Ophthalmology therefore does not separate out cleanly into primary, secondary, and tertiary sectors on the basis of the environment in which care takes place or on the basis of the professional groups contributing to the service. Blach, ${ }^{2}$ in a commentary in the $B J O$ in 2001, argued for the creation of multiprofessional "community ophthalmic teams" and suggested that the concept should be tested in a pilot study in a defined community.

One of the stated aims of the NHS plan of 2000 was to end "old fashioned demarcations between staff and barriers between services." ${ }^{\prime 3}$ The Eye Care Services Steering Group (ECSSG) was set up by ministers in 2002 to "develop proposals for the modernisation of NHS eyecare services, maintaining and developing an integrated, patient-centred service and improving access, choice, waiting times and quality for all sectors of the community." ${ }^{4}$ The first report of the ECSSG in April 2004 encourages primary care trusts (PCTs) to “...develop integrated commissioning plans in respect of ophthalmic services across primary and secondary care sectors to best meet local needs utilising the full available workforce including ophthalmologists, optometrists, dispensing opticians, orthoptists, etc." ${ }^{\prime 4}$ The report emphasises the role of community optometrists and ophthalmic medical practitioners (OMPs) in diagnosis, refinement of referrals, and treatment of some eye conditions with the implication that this will relieve pressure on the hospital eye service.

Two NHS strategy documents published during 2005 strongly reinforce this direction of travel in policy. ${ }^{5}$ PCTs will be required to give up nearly all their provider functions and strengthen their commissioning role by the end of 2006. They will be required to demonstrate contestability (that is, competition) in commissioning of secondary care services by offering to patients a choice of four or five providers, including independent sector providers. Any "accredited provider which can deliver services to NHS standards at the NHS tariff" will be eligible to apply. ${ }^{5}$ There will be a strategic shift of resources from secondary to primary care, and ophthalmology is specifically targeted as a specialty where outpatient attendances can be "safely and effectively offered in a community setting."

Although the stated aims of "Creating a Patient-led NHS" N $^{5}$ and "Commissioning a Patient-led NHS" ${ }^{\prime 6}$ are to make NHS service more responsive to the needs of patients and to increase choice, they contain an implicit assumption that hospitals are expensive, rigid, and resistant to change while primary care providers are cheaper, more flexible, and more innovative. Is this simply an Orwellian "Four legs good, two legs bad" mantra, or does ophthalmology need to move away from a hospital based model of service to thrive? Will clinical services become more innovative and responsive, or simply more fragmented? The political imperative to implement these changes appears to be so strong that there is unlikely to be much opportunity to test their effect in advance of widespread implementation and these questions are more likely to be debated by health service historians than health service planners.

With changes about to take place, which have such profound implications for patients and for the ophthalmic workforce, what should ophthalmic primary care look like? Riad et $a l^{7}$ proposed the following working definition of primary care ophthalmology:

- "...the provision of first contact care for all ophthalmic conditions and the follow-up, preventive and rehabilitative care of selected ophthalmic conditions. It can be delivered in a variety of settings and by a diverse workforce, but in strict, efficient and timely coordination, to attain the best clinical outcome possible for the patient. A service is designated as ophthalmic primary care, only if appropriately integrated with the patient's main primary care provider, in order to ensure continuity, longitudinality and comprehensiveness in the overall care of the patient. The primary care ophthalmic service itself should be accessible, equitable, knowledgeable, responsive and efficient. In these aims, it is supported by the population sciences which identify the medical and service needs of the population served."

Primary care ophthalmology is thus defined in similar terms to the generality of primary care as it is practised in the United Kingdom and elsewhere. On this basis, ophthalmologists, GPs (with or without a special interest in ophthalmology), optometrists, OMPs, community orthoptists, and ophthalmic nurses all contribute to the provision of "first contact care...," to differing degrees, in a variety of environments, but sometimes with considerable duplication of effort. Each professional group has particular and distinctive skills and it would be a mistake to view primary care ophthalmology as the exclusive province of one professional group.

The Royal College of Ophthalmologists ${ }^{8}$ recommends a medically led, multiprofessionally delivered model of primary care ophthalmology. Primary care ophthalmologists can bring to the clinical service abilities to solve complex clinical problems, work "beyond protocol" where necessary, teach, and train. These skills are essential if primary care ophthalmology is to be capable of providing "...first contact care for all eye conditions." ${ }^{17}$ Existing primary care ophthalmologists come from a variety of medical backgrounds and most have followed somewhat unconventional career paths with few precedents to guide them. There is a good case for developing a dedicated 
training programme for the next generation of primary care ophthalmologists. This could share some modules with ophthalmic surgical training, but would concentrate on developing areas such as teaching skills, epidemiology, population based research and management skills to a higher level than is usually attained in ophthalmic surgical training.

It is difficult to guess what effect the impending changes in the way clinical services are commissioned will have on the way ophthalmology is practised in the future. The need for vitreoretinal surgery, treatment for choroidal neovascularisation, neuro-ophthalmology, and other ophthalmic specialist services will continue to exist, but we cannot be sure that they will necessarily happen under the same roof. We do not know for certain whether primary care ophthalmology will take place in hospitals, treatment centres, general practice, optometric practices, or other premises. Are we moving towards a new "hub and spoke" concept where ophthalmic primary care becomes the hub and the ophthalmic specialties the spokes?

The breadth and pace of political change might mean that the concepts of ophthalmic primary care are implemented without rehearsal and without the luxury of a safety net, as hospital provision of ophthalmic primary care is scaled down. We may be travelling in the right direction, but it feels like driving without headlights. The challenge for academic ophthalmology will be to find ways of evaluating the effects of change as they occur and disseminating the learning points rapidly.

Br J Ophthalmol 2006;90:669-670.

doi: $10.1136 /$ bjo.2006.092817

Correspondence to: Richard Smith, Buckinghamshire Hospitals Trust, Stoke Mandeville Hospital, Aylesbury, HP21 8AL, UK. richard.smith@doctors.org.uk
Competing interests: none.

\section{REFERENCES}

1 Claoué C, Foss A, Cooling R. Why are new patients coming to the eye clinic? An analysis of the relative frequencies of ophthalmic disease amongst new patients attending hospital eye clinics in two separate locations. Eye 1997; 11:865-8.

2 Blach RK. The delivery of ophthalmic care: the practitioner, community ophthalmic teams and contract ophthalmology. Br J Ophthalmol 2001;85: 1274-5.

3 Department of Health. The NHS Plan: a plan for investment, a plan for reform. London: DoH, July, 2000

4 Department of Health. National Eye Care Services Steering Group-first report. London: DoH, April, 2004.

5 Department of Health. Creating a patient-led NHS-delivering the NHS Improvement Plan. London: DoH, April, 2005.

6 Department of Health. Commissioning a patientled NHS. London: DoH, July 2005.

7 Riad SF, Dart JKG, Cooling RJ. Primary care and ophthalmology in the UK. Br J Ophthalmol 2003;87:493-9.

8 Royal College of Ophthalmologists. Ophthalmic Services Directory, 2005 (www.rcophth.ac.uk/ docs/college/OphthalmicPrimaryCare2005.pdf).
Amblyopia

\section{The timing of patching treatment and a child's wellbeing}

\section{Williams, J Horwood, K Northstone, D Herrick, A Waylen, D Wolke, ALSPAC Study Group}

\section{Bullying and eye patching}

T he psychological impact that patching treatment for amblyopia might have on children and their families was highlighted in an influential review of amblyopia screening. ${ }^{1}$ A recent randomised trial investigating the efficacy of patching for amblyopia has reported that 4 year old and 5 year old children were significantly more upset by receiving patching and glasses than by receiving glasses only, as were the parents of the 4 year olds undergoing treatment, suggesting that patching treatment can be associated with some short term distress for the child and the family. ${ }^{2}$ However, most children in the study were reported as being happy and had normal behavioural scores. We have recently reported results from a birth cohort study suggesting that longer term psychosocial sequelae may be associated with patching treatment (the Avon Longitudinal Study of Parents and Children, ALSPAC ${ }^{3}$ ). A history of patching treatment and wearing glasses were both independent risk factors for children reporting that they had been bullied in the past at age 8. ${ }^{4}$ Repeated bullying victimisation is consistently associated with physical and emotional problems for the victims and may have long term adverse consequences. $^{56}$

One argument for preschool screening is that patching treatment is more likely to have concluded before school starts, thus avoiding adverse reactions from peers. We aimed to test this hypothesis by comparing, prospectively, two groups of children: one that had been offered state provided preschool screening for amblyopia (aged 3 years and 1 month) and the other that had not. Both groups had been given a check by the school nurse in the first year of school (aged between 4-5), which if unsatisfactory resulted in a recommendation to see either an optometrist and/or a community orthoptist, after which patching was commenced if needed. The outcome measure was bullying victimisation by age 8 assessed with a structured standard interview. ${ }^{6}$ Children were asked whether they had repeatedly (at least four times a month) been the victims of bullying. ${ }^{4}$ We calculated the risk (odds ratio, $95 \%$ confidence interval) of reporting having been bullied for children who had been treated with patching in each group. For comparison, we calculated the same risk for children who had been given glasses at any time. We hypothesised that as the wearing of glasses usually continues once started, then preschool screening would be unlikely to reduce any risk of bullying associated with wearing glasses.

Usable data on having been bullied were available for 4473 children whose screening history was known and these are shown in table 1. There was an almost $50 \%$ reduction in children who reported having been bullied in the group that had been offered preschool screening, compared with the group who had not. By contrast, there was no difference in rates of perceived bullying for children who wore glasses, irrespective of previous screening. These results support the hypothesis that preschool vision screening may be associated with less bullying for children who need to have patching treatment. The specificity of the findings (that is, there was no such effect for children who wore glasses) argues against the results being the result of confounding factors that have not been accounted for, although as the data are observational, this possibility cannot be excluded. 